\title{
Adiponectin affects vascular smooth muscle cell proliferation and apoptosis through modulation of the mitofusin-2-mediated Ras-Raf-Erk1/2 signaling pathway
}

\author{
WENBO ZHANG, CHANG SHU, QUANMING LI, MING LI and XIN LI \\ Department of Vascular Surgery, The Second Xiangya Hospital of Central South University, \\ Changsha, Hunan 410011, P.R. China
}

Received August 13, 2014; Accepted May 6, 2015

DOI: $10.3892 / \mathrm{mmr} .2015 .3899$

\begin{abstract}
Vascular smooth muscle cells (VSMCs) undergo cell biological changes in response to a variety of cytokines and growth factors. Adiponectin inhibits neointimal formation through suppressing the proliferation and migration of VSMCs. However, the mechanisms underlying the effect of adiponectin on VSMC proliferation and apoptosis require further investigation. The present study was designed to investigate the mechanisms of adiponectin on VSMC proliferation and apoptosis, focusing on the mitofusin-2 (MFN2) mediated Ras-Raf-extracellular signal regulated kinase (Erk)1/2 signaling pathway. The results of western blot analysis revealed that adiponectin increased the expression of MFN2 in a concentration-dependent manner. Adiponectin also suppressed VSMC proliferation and induced VSMC apoptosis. However, transfection of the VSMCs with small interfering (si)RNA, to knock down the expression of MFN2 attenuated the effect of adiponectin on VSMC proliferation and apoptosis. The decreased expression levels of Ras, phosphorlated (p)-c-Raf and p-Erk1/2, observed in the VSMCs treated with adiponectin were also reversed by the transfection of the VSMCs with MFN2 siRNA to knock down the expression of MFN2. The results of the present study demonstrated for the first time, to the best of our knowledge, that adiponectin exhibits an inhibitory effect on VSMC proliferation and induces cell proliferation via regulation of the expression levels of MFN2. Adiponectin upregulated the expression of MFN2, inhibiting the Ras-Raf-Erk1/2 signaling pathway, which led to the inhibition of VSMC proliferation and the induction of VSMC apoptosis. The results of the
\end{abstract}

Correspondence to: Professor Chang Shu, Department of Vascular Surgery, The Second Xiangya Hospital of Central South University, 139 Middle Renming Road, Changsha, Hunan 410011, P.R. China E-mail: changshu655@163.com

Key words: adiponectin, mitofusin-2, vascular smooth muscle cells, Ras-Raf-Erk1/2 signaling pathway present study may provide a novel basis for the therapy of vascular disease.

\section{Introduction}

Vascular smooth muscle cells (VSMCs) are an important constituent of vessel walls, and their dysfunction may lead to pathological processes and cause vascular disease. VSMCs undergo cell biological changes in response to a variety of cytokines and growth factors (1).

Adiponectin is an important adipocyte-derived hormone (2,3), which is well-known for its involvement in lipid and glucose metabolism and insulin sensitivity (4-7). Numerous studies have suggested that adiponectin has potent anti-inflammatory (8), athero-protective (9), antihypertensive (10) and antidiabetic (11) effects. A previous study demonstrated that adiponectin is also expressed and secreted by VSMCs (12), and adiponectin inhibits neointimal formation through suppression of the proliferation and migration of VSMCs (13-15). However, the mechanisms underlying the effect of adiponectin on VSMC proliferation and apoptosis require further investigation.

The present study aimed to investigate whether mitofusin-2 (MFN2) which mediates the effect of adiponectin on VSMC proliferation and apoptosis, which may provide a novel basis for the therapy of vascular disease.

\section{Materials and methods}

Cell culture. The Aortic Smooth Mus cells (VSMCs; cat. no. CC-2571) were purchased from Clonetics (San Diego, CA, USA). The VSMCs were cultured in Dulbecco's modified Eagle's medium (Gibco-BRL, Rockville, MD, USA) with $20 \%$ fetal bovine serum (Gibco-BRL) in a humidified atmosphere at $37^{\circ} \mathrm{C}$ of $5 \% \mathrm{CO}_{2}$. The culture medium was replaced every 3 days.

Treatment of VSMCs with adiponectin. Adiponectin was purchased from Cayman Chemical Company (Ann Arbor, MI, USA) and dissolved in phosphate-buffered saline (1 $\mathrm{mg} / \mathrm{ml})$. To obtain different experimental concentrations $(5,10,20$ and $40 \mu \mathrm{g} / \mathrm{ml})$, serial dilutions were prepared in the 
culture medium. VSMCs $\left(5 \times 10^{5}\right.$ cells $\left./ \mathrm{ml}\right)$ were seeded into 6-well plates and when the VSMCs reached $80 \%$ confluence, adiponectin was added to treat the cells for $24 \mathrm{~h}$ at $37^{\circ} \mathrm{C}$.

Transfection. The cells were seeded into 6-well plates at a density of $3 \times 10^{5}$ cells/well and allowed to grow for $24 \mathrm{~h}$. MFN2 small interfering (si)RNA, forward 5'-CCAUGAGGCCUU UCUCCTT-3' and reverse 5'-GGAGAAAGGCCUCAUGGT T-3'; or control siRNA, forward 5'-UUCUCCGAACGUGUC ACGUTT-3' and reverse 5'-ACGUGACACGUUCGGAGA ATT-3'. were synthesized at Sangon Biotech Inc. (Shanghai, China) and transfected into the cells at a final concentration of $2 \mu \mathrm{M}$ using lipofectamine 2000 transfection reagent (Invitrogen Life Technologies, Carlsbad, CA, USA), according to the manufacturer's instructions.

Flow cytometry assay. For cell apoptosis analysis using flow cytometry, the cells were harvested and fixed in 70\% ethanol (Xinyu Inc., Shanghai, China) on ice. Following centrifugation at $1,000 \mathrm{x}$ g for $5 \mathrm{~min}$ at room temperature, the cells were stained with annexin $\mathrm{V}$ and propidium iodide (Kaiji Biological Inc., Nanjing, China), according to the manufacturer's instructions, and analyzed using flow cytometry (FC 500 MPL system; Beckman Coulter, Inc., Miami, FL, USA).

MTT assay. The cells were seeded into 96-well plates at a density of $4 \times 10^{3}$ cells/well. Following treatment with adiponectin for 24, 48 and $72 \mathrm{~h}, 10 \mu \mathrm{l}$ MTT solution (Sigma-Aldrich, St. Louis, MO, USA) was added to each well, and the incubation was continued for $4 \mathrm{~h}$ at $37^{\circ} \mathrm{C}$. The formazan was solubilized in dimethyl sulfoxide (Sigma-Aldrich) and the viable cells were determined by measuring the absorbance at $570 \mathrm{~nm}$ using a microplate reader (Multiskan Ascent 354; Thermo Labsystems, Waltham, MA, USA).

Western blot analysis. The proteins from cultured cells were extracted using cell lysis buffer, containing $50 \mathrm{mM}$ Tris (Amresco LLC, Solon, OH, USA), $150 \mathrm{mM} \mathrm{NaCl}$ (Tianjin Dingshengxin Chemical Industry Co., Ltd., Tianjin, China), 1 mM EDTA (Zhiyuan, Inc., Tianjin, China), $1 \%$ Triton X-100 (Amresco LLC), $1 \mathrm{mM}$ sodium orthovanadate (Beyotime Institute of Biotechnology, Shanghai, China), $1 \mathrm{mM}$ PMSF (Amresco LLC) and 2 mM DTT (pH 7.4; Amresco LLC). Protein concentrations were quantified using a bicinchoninic acid assay method, using reagents purchased from Pierce Biotechnology, Inc. (Rockford, IL, USA). Equal quantities of the cell lysates were separated on 12\% SDS-PAGE (Invitrogen Life Technologies, Carlsbad, CA, USA), and then transferred on to polyvinylidene difluoride membranes (Millipore, Billerica, MA, USA) via electroblotting. The membranes were blocked with Tris-buffered saline containing $5 \%$ bovine serum albumin (Sigma-Aldrich) at $37^{\circ} \mathrm{C}$ for $1 \mathrm{~h}$. The blot was then probed with mouse monoclonal antibody against MFN2 (sc-100560; 1:500; Santa Cruz Biotechnology, Inc., Santa Cruz, CA, USA), mouse monoclonal antibody against Ras (sc-166691; 1:800; Santa Cruz Biotechnology, Inc.), mouse monoclonal antibody against c-Raf (\#12552; 1:400; Cell Signaling Technology, Inc., Beverly, MA, USA), rabbit monoclonal antibody against extracellular signal regulated kinase (Erk)1/2 (\#4695; 1:400; Cell Signaling Technology, Inc.), rabbit monoclonal antibody against phosphorylated (p)-c-Raf (Ser338; \#9427; 1:400; Cell Signaling Technology, Inc.) and rabbit monoclonal antibody against p-Erk1/2 (Thr202/Tyr204; \#4377; 1:400; Cell Signaling Technology, Inc.). Subsequently, the membranes were incubated with horseradish peroxidase (HRP)-conjugated secondary antibody [goat anti-rabbit $\mathrm{IgG} / \mathrm{HRP}(\mathrm{sc}-2004 ; 1: 10,000)$ and goat anti-mouse IgG/HRP (sc-2031; 1:10,000); Santa Cruz Biotechnology, Inc.)] at $37^{\circ} \mathrm{C}$ for $1 \mathrm{~h}$. The signals were detected using an enhanced chemiluminescence western blotting kit (Pierce Biotechnology, Inc).

Statistical analysis. All data are expressed as the mean \pm standard deviation of at least three independent experiments. Comparisons of the parameters between the two groups were performed using Student's t-test. SPSS software, version 19 (IBM SPSS, Armonk, NY, USA) was used for statistical analysis. $\mathrm{P}<0.05$ was considered to indicate a statistically significant difference.

\section{Results}

Adiponectin induces the expression of MFN2 in VSMCs. To investigate the effect of adiponectin on the expression of MFN2, the VSMCs were treated with adiponectin at concentrations between 5 and $40 \mu \mathrm{g} / \mathrm{ml}$, and, after $24 \mathrm{~h}$, the expression of MFN2 in the VSMCs was assessed using western blot analysis. The results are shown in Fig. 1, and revealed that the expression of MFN2 increased with increasing concentrations of adiponectin. Concentrations between 10 and $40 \mu \mathrm{g} / \mathrm{ml}$ adiponectin significantly increased the expression of MFN2, with the peak value observed at $40 \mu \mathrm{g} / \mathrm{ml}(\mathrm{P}<0.05)$.

Inhibition of the Ras-Raf-Erk1/2 signaling pathway following treatment with adiponectin. The Ras-Raf-Erk1/2 pathway is one of the most important downstream signaling cascades of MFN2. The effect of adiponectin on the expression levels of Ras, p-c-Raf and p-Erk1/2 was investigated in the present study. The relative expression level of Ras was normalized to that of $\beta$-actin, while the relative expression levels of the phosphorylated forms of c-Raf and Erk1/2 were normalized to the levels of total c-Raf and Erk1/2. The western blot analysis revealed that the relative expression levels of Ras, p-c-Raf, p-Erk1/2 in the VSMCs were significantly inhibited by adiponectin at concentrations between 10 and $40 \mu \mathrm{g} / \mathrm{ml}$ $(\mathrm{P}<0.05)$ (Fig. 2).

MFN2 knockdown attenuates the inhibitory effect of adiponectin on VSMC proliferation. To examine whether MFN2 was involved in the effect of adiponectin on VSMC proliferation, siRNA-MFN2 was transfected into VSMCs for $24 \mathrm{~h}$ to knock down the expression of MFN2, following which the VSMCs were treated with $40 \mu \mathrm{g} / \mathrm{ml}$ adiponectin for another $24 \mathrm{~h}$. The results revealed that $40 \mu \mathrm{g} / \mathrm{ml}$ adiponectin upregulated the expression of MFN2 in the VSMCs $(\mathrm{P}<0.05)$. However, the expression ofMFN2 was suppressed by transfection with siRNA-MFN2, with the expression of MFN2 significantly decreased in the adiponectin + siRNA-MFN2 group, compared with the adiponectin + siRNA-control group $(\mathrm{P}<0.01$; Fig. 3$)$. 

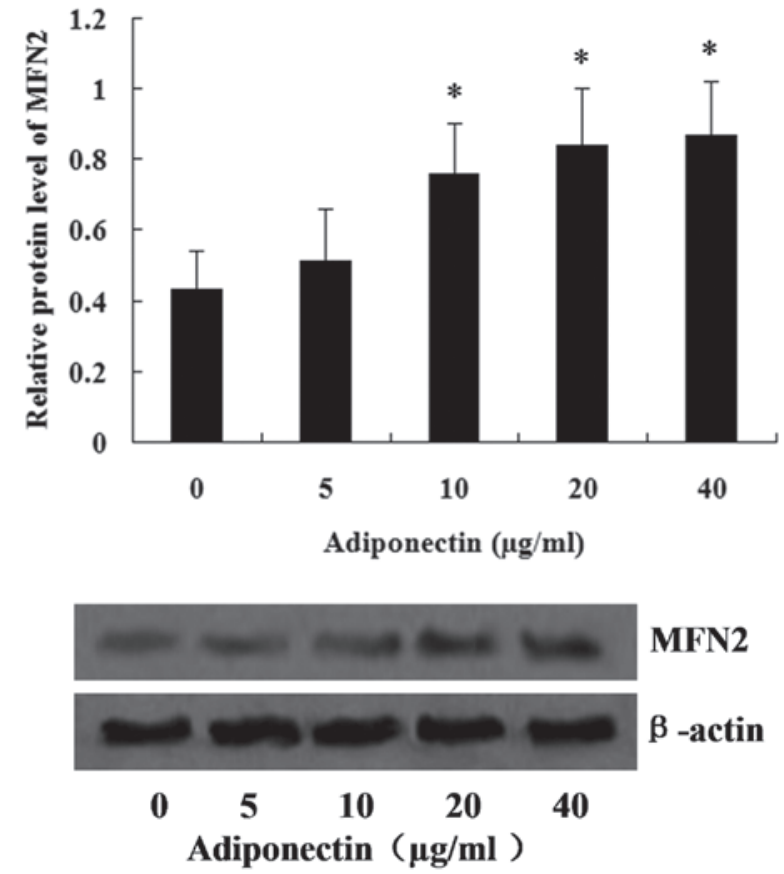

Figure 1. Effect of adiponectin on the expression of MFN2 using western blotting. The relative expression level of MFN2 was normalized to $\beta$-actin. Data are expressed as the mean \pm standard deviation. ${ }^{*} \mathrm{P}<0.05$, compared with $0 \mu \mathrm{g} / \mathrm{ml}$. MFN2, mitofusin-2.

An MTT assay was used to examine VSMC proliferation. As shown in Fig. 4, the VSMC survival rates decreased significantly following treatment with adiponectin $(\mathrm{P}<0.05)$. However, MFN2-knockdown attenuated the inhibitory effect of adiponectin on VSMC proliferation, and the VSMC survival rates were significantly increased in the adiponectin + siRNA-MFN2 group, compared with the adiponectin + siRNA-control group $(\mathrm{P}<0.05$; Fig. 4$)$.

MFN2 knockdown attenuates adiponectin-induced apoptosis in VSMCs. Subsequently, the present study examined whether MFN2 was involved in the effect of adiponectin on VSMC apoptosis. The VSMCs were transfected either with siRNA-MFN2 or siRNA-control for $24 \mathrm{~h}$, followed by treatment with $40 \mu \mathrm{g} / \mathrm{ml}$ adiponectin for another $24 \mathrm{~h}$. Subsequently, the VSMCs were harvested for flow cytometric analysis. As shown in Fig. 5, the apoptotic rate of the cells in the control group was $8.3 \pm 1.6 \%$, whilst treatment with adiponectin significantly induced cell apoptotic rates to $15.2 \pm 2.7 \%(\mathrm{P}<0.05)$. The apoptotic rate of the cells in the adiponectin + siRNA-MFN2 group $(9.5 \pm 2.1 \%)$ was significantly different from the adiponectin + siRNA-control group $(15.2 \pm 2.7 \%)(\mathrm{P}<0.05)$.

MFN2 knockdown attenuates the inhibitory effect of adiponectin on the Ras-Raf-Erk1/2 signaling pathway. Western blot analysis was used to assess the role of MFN2-knockdown in the inhibitory effect of adiponectin on the Ras-Raf-Erk1/2 signaling pathway. As shown in Fig. 6, adiponectin significantly inhibited the relative expression levels of Ras $(\mathrm{P}<0.05)$, p-c-Raf $(\mathrm{P}<0.01)$ and $\mathrm{p}-\mathrm{Erk} 1 / 2(\mathrm{P}<0.01)$ in the VSMCs. However, MFN2-knockdown attenuated the inhibitory effect of adiponectin, and the relative expression levels of Ras,
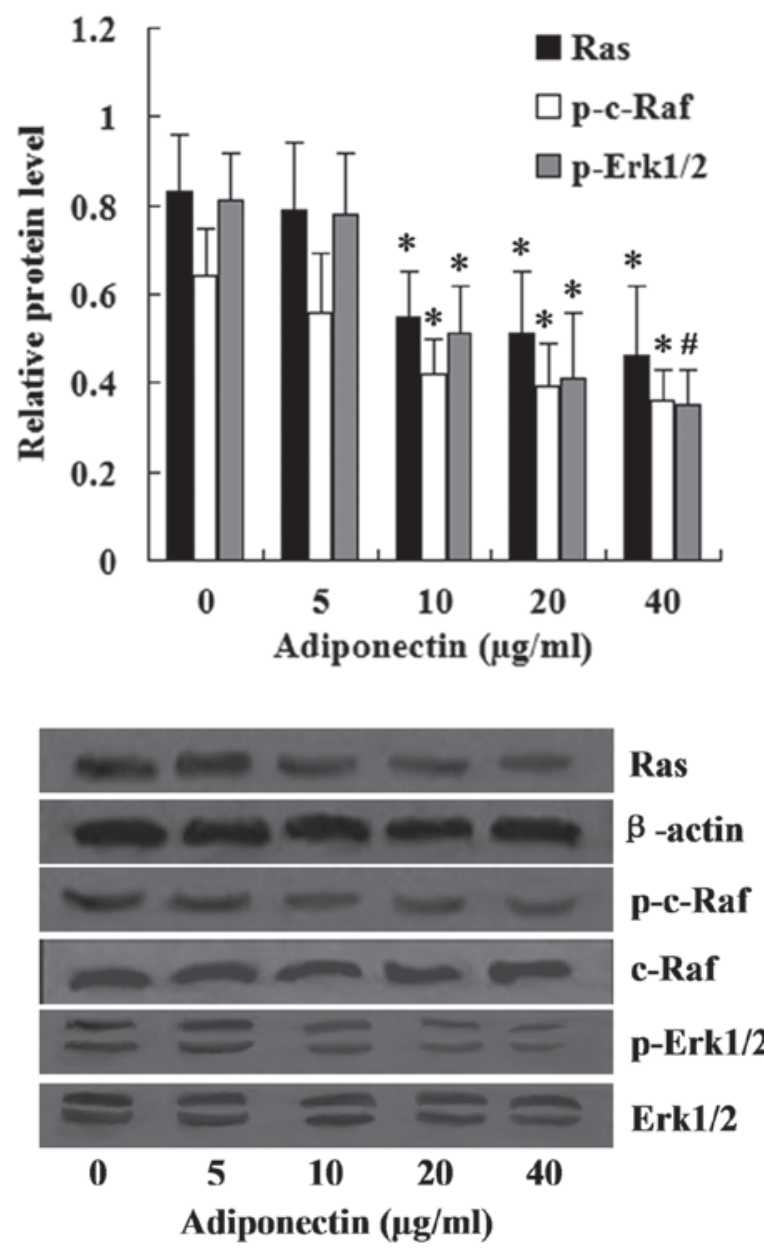

Figure 2. Effect of adiponectin on the expression levels of Ras, p-c-Raf and p-Erk1/2. The relative expression level of Ras was normalized to $\beta$-actin while the relative expression levels of $\mathrm{p}-\mathrm{c}-\mathrm{R}$ af and $\mathrm{p}$-Erk1/2 were normalized to total c-Raf and Erk1/2. Data are expressed as the mean \pm standard deviation. ${ }^{*} \mathrm{P}<0.05$ and ${ }^{\#} \mathrm{P}<0.01$, compared with $0 \mu \mathrm{g} / \mathrm{ml}$. ERK, extracellular signal-regulated kinase; p-, phosphorylated.

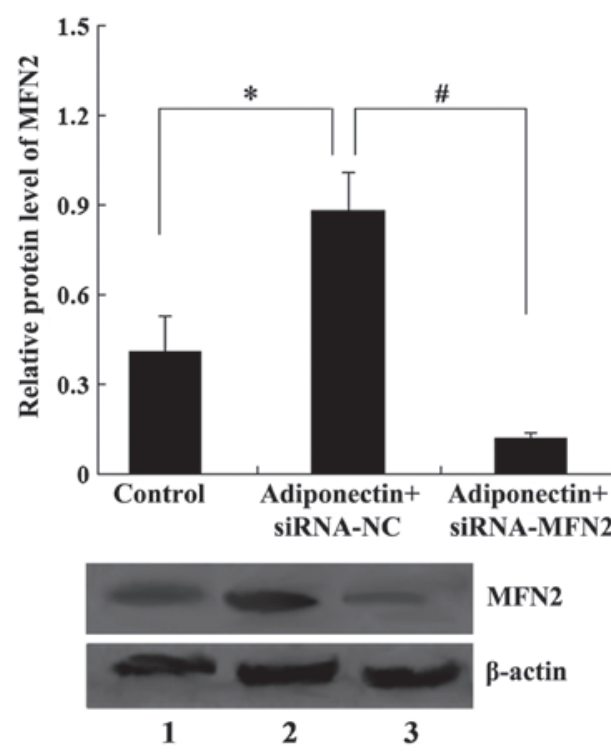

Figure 3. Relative expression level of MFN2 in VSMCs transfected with siRNA. Lane 1, control; lane 2, adiponectin + siRNA-NC; lane 3, adiponectin + siRNA-MFN2. Data are expressed as the mean \pm standard deviation. ${ }^{*} \mathrm{P}<0.05$ and ${ }^{\#} \mathrm{P}<0.01$. VSMC, vascular smooth muscle cell; siRNA, small interfering RNA; MFN2, mitofusin-2; NC, negative control. 


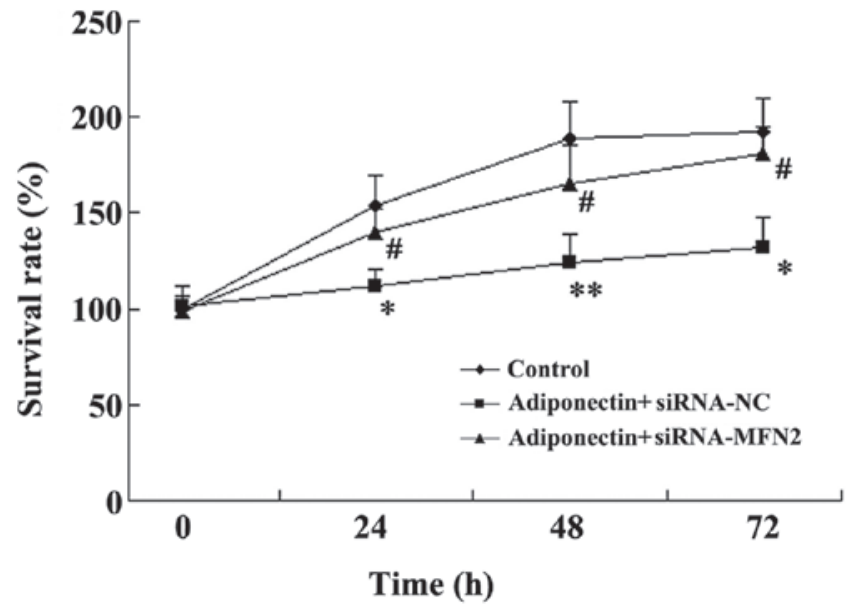

Figure 4. MFN2 knockdown attenuates the inhibitory effect of adiponectin on VSMC proliferation. Data are expressed as the mean \pm standard deviation. ${ }^{*} \mathrm{P}<0.05$ and ${ }^{* *} \mathrm{P}<0.01$, compared with the control group; ${ }^{*} \mathrm{P}<0.05$, compared with the adiponectin+siRNA-NC group. VSMC, vascular smooth muscle cell; siRNA, small interfering RNA; MFN2, mitofusin-2; NC, negative control.

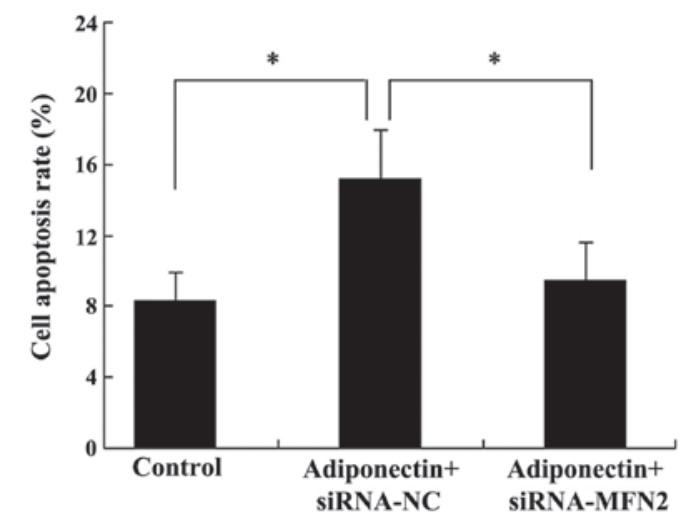

Figure 5. MFN2 knockdown attenuates adiponectin-induced VSMC apoptosis. 1, control group; 2, adiponectin + siRNA-NC group; 3, adiponectin + siRNA-MFN2 group. Data are expressed as the mean \pm standard deviation. ${ }^{*} \mathrm{P}<0.05$. VSMC, vascular smooth muscle cell; siRNA, small interfering RNA; MFN2, mitofusin-2; NC, negative control.

p-c-Raf and p-Erk1/2 were significantly increased in the adiponectin + siRNA-MFN2 group, compared with the adiponectin + siRNA-control group $(\mathrm{P}<0.05)$.

\section{Discussion}

It has been previously demonstrated that adiponectin is involved in the protection of vascular injury by suppressing neointimal formation $(16,17)$. The present study demonstrated that adiponectin exhibited an inhibitory effect on VSMC proliferation and induced cell apoptosis, and these findings were consistent with those of previous studies $(13-15,18)$.

As mentioned previously, certain genes and signaling pathways have been implicated in the effect of adiponectin on cell growth (19-22). In the present study, MFN2 and its downstream signaling cascade, the Ras-Raf-Erk1/2 signaling pathway, were investigated.

MFN2 is a mitochondrial membrane protein, which is primarily involved in mitochondrial fusion (23). MFN2 acts
A

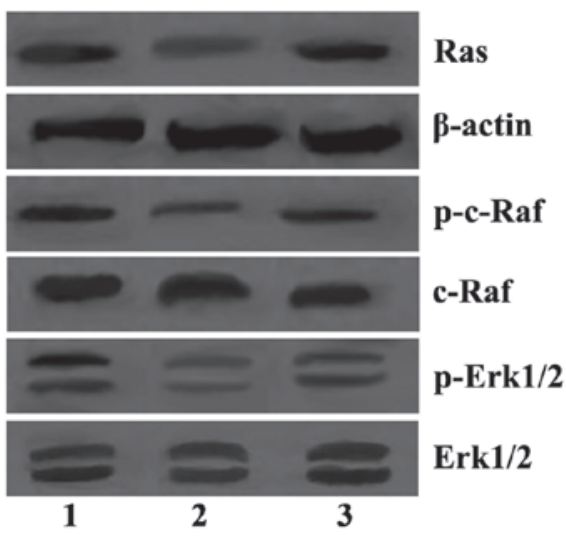

B

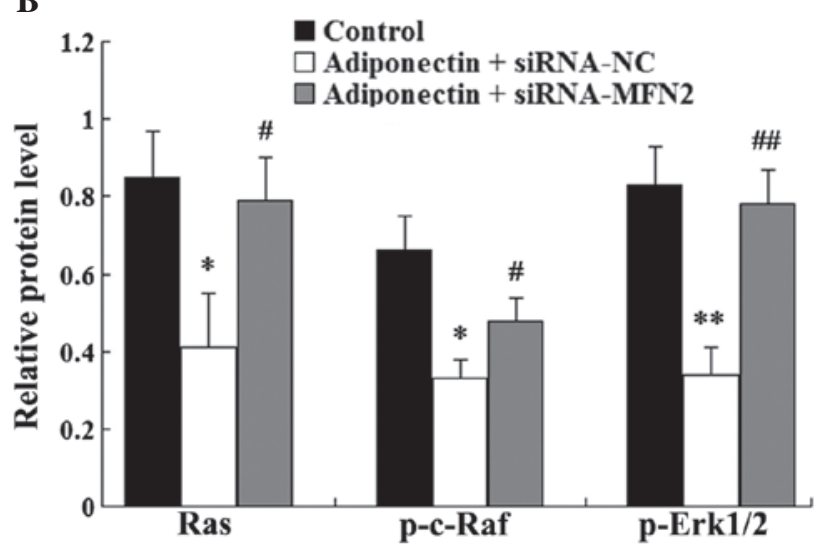

Figure 6. MFN2 knockdown attenuates the inhibitory effect of adiponectin on the Ras-Raf-Erk1/2 signaling pathway. (A) Western blot analysis of Ras, c-Raf, Erk1/2, p-c-Raf and p-Erk1/2 in the VSMCs. Lane 1, control group; lane 2, adiponectin + siRNA-NC group; lane 3, adiponectin + siRNA-MFN2 group. (B) Relative protein levels of Ras, p-c-Raf and p-Erk1/2. The relative expression level of Ras was normalized to $\beta$-actin, while the relative expression levels of p-c-Raf and p-Erk1/2 were normalized to total c-Raf and Erk1/2. Data are expressed as the mean \pm standard deviation. ${ }^{*} \mathrm{P}<0.05$ and ${ }^{* *} \mathrm{P}<0.01$, compared with the control group; ${ }^{\#} \mathrm{P}<0.05$ and ${ }^{\# \#} \mathrm{P}<0.01$, compared with the adiponectin + siRNA-NC group. VSMC, vascular smooth muscle cell; siRNA, small interfering RNA; MFN2, mitofusin-2; NC, negative control; ERK, extracellular signal-regulated kinase; p-, phosphorylated.

as a suppressor in cell proliferation and as a promoter of cell apoptosis (24-28). It was revealed in the present study that siRNA-mediated downregulation of MFN2 in the VSMCs attenuated the effect of adiponectin on VSMC proliferation and apoptosis, therefore the results demonstrated that MFN2 was involved in the effect of adiponectin on VSMC proliferation and apoptosis. In addition, it was revealed that adiponectin significantly increased the expression of MFN2 in a concentration-dependent manner.

Previous studies have indicated that the Ras-Raf-Erk1/2 signaling pathway is the downstream signaling cascade of MFN2 (29,30). Following activation of Ras, it couples with Raf directly and the cellular Raf protein is phosphorylated, the downstream signaling transduction pathways are then activated, leading to the phosphorylation of Erk1 and Erk2. The activated Erk1/2 protein contributes to the growth of VSMCs $(31,32)$. However, MFN2 acts as a negative regulator of the Ras-Raf-Erk1/2 signaling pathway through its interaction with Ras (29). In the present study, the results demonstrated that, in the VSMCs, adiponectin significantly inhibited the 
expression levels of Ras, p-c-Raf and p-Erk1/2, however, MFN2 knockdown attenuated the inhibitory effect of adiponectin. This demonstrated that MFN2 mediated the effects of adiponectin on the Ras-Raf-Erk1/2 signaling pathway.

In conclusion, the present study demonstrated for the first time, to the best of our knowledge, that adiponectin exhibited an inhibitory effect on VSMC proliferation, and induced cell proliferation via regulation of the expression of MFN2. Adiponectin also upregulated the expression of MFN2, which inhibited the Ras-Raf-Erk1/2 signaling pathway, leading to the inhibition of VSMC proliferation and to the induction of VSMC apoptosis. The results of the present study improves and expands the current understanding of the effects of adiponectin on VSMCs and may provide novel targets for therapeutic intervention in vascular disease.

\section{References}

1. Kiyan Y, Limbourg A, Kiyan R, et al: Urokinase receptor associates with myocardin to control vascular smooth muscle cells phenotype in vascular disease. Arterioscler Thromb Vasc Biol 32: 110-122, 2012.

2. Matsuzawa Y, Funahashi $\mathrm{T}$ and Nakamura T: Molecular mechanism of metabolic syndrome X: contribution of adipocytokines adipocyte-derived bioactive substances. Ann N Y Acad Sci 892: 146-154, 1999.

3. Henry BA and Clarke IJ: Adipose tissue hormones and the regulation of food intake. J Neuroendocrinol 20: 842-849, 2008.

4. Yamauchi T, Kamon J, Waki H, et al: The fat-derived hormone adiponectin reverses insulin resistance associated with both lipoatrophy and obesity. Nat Med 7: 941-946, 2001.

5. Berg AH, Combs TP, Du X, Brownlee M and Scherer PE: The adipocyte-secreted protein Acrp30 enhances hepatic insulin action. Nat Med 7: 947-953, 2001.

6. Maeda N, Shimomura I, Kishida K, et al: Diet-induced insulin resistance in mice lacking adiponectin/ACRP30. Nat Med 8: 731-737, 2002.

7. Matsuzawa Y: Establishment of a concept of visceral fat syndrome and discovery of adiponectin. Proc Jpn Acad Ser B Phys Biol Sci 86: 131-141, 2010.

8. Wulster-Radcliffe MC, Ajuwon KM, Wang J, Christian JA and Spurlock ME: Adiponectin differentially regulates cytokines in porcine macrophages. Biochem Biophys Res Commun 316: 924-929, 2004.

9. Matsuda M, Shimomura I, Sata M, et al: Role of adiponectin in preventing vascular stenosis. The missing link of adipo-vascular axis. J Biol Chem 277: 37487-37491, 2002.

10. Chen H, Montagnani M, Funahashi T, Shimomura I and Quon MJ: Adiponectin stimulates production of nitric oxide in vascular endothelial cells. J Biol Chem 278: 45021-45026, 2003.

11. Maeda N, Shimomura I, Kishida K, et al: Dietinduced insulin resistance in mice lacking adiponectin/ACRP30. Nat Med 8: 731-737, 2002.

12. Ding M, Carrão AC, Wagner RJ, et al: Vascular smooth muscle cell-derived adiponectin: a paracrine regulator of contractile phenotype. J Mol Cell Cardiol 52: 474-484, 2012.

13. Matsuda M, Shimomura I, Sata M, et al: Role of adiponectin in preventing vascular stenosis. The missing link of adipo-vascular axis. J Biol Chem 277: 37487-37491, 2002.
14. Wang Y, Lam KS, Xu JY, Lu G, Xu LY, Cooper GJ and Xu A: Adiponectin inhibits cell proliferation by interacting with several growth factors in an oligomerization-dependent manner. J Biol Chem 280: 18341-18347, 2005.

15. Motobayashi Y,Izawa-Ishizawa Y, Ishizawa K, et al: Adiponectin inhibits insulin-like growth factor-1-induced cell migration by the suppression of extracellular signal-regulated kinase 1/2 activation, but not Akt in vascular smooth muscle cells. Hypertens Res 32: 188-193, 2009.

16. Takaoka M, Nagata D, Kihara S, et al: Periadventitial adipose tissue plays a critical role in vascular remodeling. Circ Res 105: 906-911, 2009.

17. Miao CY and Li ZY: The role of perivascular adipose tissue in vascular smooth muscle cell growth. $\mathrm{Br}$ J Pharmacol 165: 643-658, 2012.

18. Nepal S and Park PH: Regulatory role of autophagy in globular adiponectin-induced apoptosis in cancer cells. Biomol Ther (Seoul) 22: 384-389, 2014.

19. Yamauchi T, Kamon J, Ito Y, et al: Cloning of adiponectin receptors that mediate antidiabetic metabolic effects. Nature 423: 762-769, 2003.

20. Hug C, Wang J, Ahmad NS, Bogan JS, Tsao TS and Lodish HF: T-cadherin is a receptor for hexameric and high-molecular-weight forms of Acrp30/adiponectin. Proc Natl Acad Sci USA 101: 10308-10313, 2004.

21. Zhou L, Deepa SS, Etzler JC, et al: Adiponectin activates AMP-activated protein kinase in muscle cells via APPL1/LKB1-dependent and phospholipase $\mathrm{C} / \mathrm{Ca} 2+/ \mathrm{Ca} 2+/ \mathrm{calmodulin}-\mathrm{dependent}$ protein kinase kinase-dependent pathways. J Biol Chem 284: 22426-22435, 2009.

22. Iwabu M, Yamauchi T, Okada-Iwabu M, et al: Adiponectin and AdipoR1 regulate PGC-1alpha and mitochondria by $\mathrm{Ca}(2+)$ and AMPK/SIRT1. Nature 464: 1313-1319, 2010.

23. Santel A and Fuller MT: Control of mitochondrial morphology by a human mitofusin. J Cell Sci 114: 867-874, 2001

24. Chen KH, Guo X, Ma D, et al: Dysregulation of HSG triggers vascular proliferative disorders. Nat Cell Biol 6: 872-883, 2004.

25. Jin B, Fu G, Pan H, et al: Anti-tumour efficacy of mitofusin-2 in urinary bladder carcinoma. Med Oncol 28 (Suppl 1): 373-380, 2011.

26. Wu L, Li Z, Zhang Y, et al: Adenovirus-expressed human hyperplasia suppressor gene induces apoptosis in cancer cells. Mol Cancer Ther 7: 222-232, 2008.

27. Wang W, Zhu F, Wang S, et al: HSG provides antitumor efficacy on hepatocellular carcinoma both in vitro and in vivo. Oncol Rep 24: 183-188, 2010.

28. Wang W, Lu J, Zhu F, et al: Pro-apoptotic and anti-proliferative effects of mitofusin-2 via Bax signaling in hepatocellular carcinoma cells. Med Oncol 29: 70-76, 2010.

29. Chen KH, Dasgupta A, Ding J, Indig FE, Ghosh P and Longo DL: Role of mitofusin 2 (Mfn2) in controlling cellular proliferation. FASEB J 28: 382-394, 2014.

30. Zhang GE, Jin HL, Lin XK, Chen C, Liu XS, Zhang Q and Yu JR: Anti-tumor effects of $\mathrm{mfn} 2$ in gastric cancer. Int J Mol Sci 14: 13005-13021, 2013.

31. Ortmann J, Veit M, Zingg S, et al: Estrogen receptor- $\alpha$ but not $\beta$ or GPER inhibits high glucose-induced human VSMC proliferation: potential role of ROS and ERK. J Clin Endocrinol Metab 96: 220-228, 2011.

32. Sirois MG, Simons M and Edelman ER: Antisense oligonucleotide inhibition of PDGFR-beta receptor subunit expression directs suppression of intimal thickening. Circulation 95: 669-676, 1997. 